\title{
BMJ Open Quality Introducing an AKI predictive tool for patients undergoing orthopaedic surgery
}

\author{
David Paul Baird, ${ }^{1}$ Fraser Rae, ${ }^{2}$ Christina Beecroft, ${ }^{3}$ Katherine Gallagher, ${ }^{4}$ \\ Stephanie Sim, ${ }^{5}$ Robert Vaessen, ${ }^{5}$ Emily Wright, ${ }^{1}$ Samira Bell ${ }^{1,6}$
}

To cite: Baird DP, Rae F, Beecroft C, et al. Introducing an AKI predictive tool for patients undergoing orthopaedic surgery. BMJ Open Quality 2019;8:e000306. doi:10.1136/ bmjoq-2017-000306

- Additional material is published online only. To view please visit the journal online (http://dx.doi.org/10.1136/ bmjoq-2017-000306)

Received 22 December 2017 Revised 17 February 2019 Accepted 26 February 2019

A Check for updates

C Author(s) (or their employer(s)) 2019. Re-use permitted under CC BY-NC. No commercial re-use. See rights and permissions. Published by BMJ.

${ }^{1}$ Renal Medicine, Ninewells Hospital, Dundee, UK

${ }^{2}$ Orthopaedic Department, Perth Royal Infirmary, Perth, Perth and Kinross, UK

${ }^{3}$ Anaesthetic Department, Ninewells Hospital, Dundee, UK ${ }^{4}$ Department of Medicine, Perth Royal Infirmary, Perth, Perth and Kinross, UK

${ }^{5}$ Anaesthetic Department, Perth Royal Infirmary, Perth, Perth and Kinross, UK

${ }^{6}$ Division of Population Health and Genomics, University of Dundee, Dundee, UK

Correspondence to Dr David Paul Baird; d.baird1@nhs.net

\section{ABSTRACT}

Patients undergoing surgery are at increased risk of acute kidney injury (AKI). AKI is associated with adverse outcomes such as increased mortality and future risk of developing chronic kidney disease. We have developed a validated preoperative scoring tool to predict postoperative AKI in patients undergoing orthopaedic surgery using seven readily available parameters. The aim of this project was to establish the use of this scoring tool with a target compliance of $80 \%$ in patients undergoing orthopaedic surgery requiring an overnight stay at Perth Royal Infirmary, a district general hospital in NHS Tayside. We created an intervention bundle for patients at high risk of AKI, which we defined as greater than $10 \%$. An electronic tool available on smartphones and desktop computers was developed that can be used to calculate the score. The interventions were incorporated into the electronic tool and posters outlining the intervention were placed in clinical areas. Patients undergoing elective procedures were scored in the preassessment clinic while emergency patients were scored by the admitting doctors. The score was introduced using four PDSA cycles. This confirmed that the scoring tool functioned well and was being used accurately. Compliance for patients undergoing elective surgery was reasonable at $19 / 24(79 \%)$ in the third and fourth PDSA cycles but was poorer for emergency admissions with compliance of only $3 / 7(43 \%)$. There was excellent compliance with the suggested medication changes and postoperative blood test monitoring as advised by our intervention bundle for those at high risk of AKI. Fluid balance monitoring was advised for all patients but the outcome was similar following our intervention at $27 / 41$ (66\%) compared with 23/37 (62\%) in the baseline data collection. Compliance with fluid balance monitoring was higher in patients at high risk of AKI (9/12, 75\%).

\section{PROBLEM}

Acute kidney injury (AKI) is an abrupt (hours to days) decrease in renal function. Patients undergoing surgery are at risk of developing an AKI postoperatively with significantly increased morbidity and mortality. Prevention of AKI is particularly important as no specific treatment, other than supportive care, is available.

In 2015, we published a preoperative scoring tool designed for patients undergoing orthopaedic surgery that predicted the risk of developing postoperative AKI. ${ }^{1}$ The tool uses seven predictors that are readily available; age, gender, presence or absence of diabetes, estimated glomerular filtration rate (eGFR), American Society of Anaesthesiologists (ASA) grade, use of ACE inhibitors or angiotensin receptor blockers (ARBs) and the number of prescribed medications. This allows identification of patients who are at high risk of AKI.

NHS Tayside is a Scottish health board which covers a population of 415000 and carries out approximately 4000 orthopaedic operations per year across three sites. The Perth Royal Infirmary carries out approximately 1100 of these operations, of which $50 \%$ are elective. It has two orthopaedic wards; an elective ward and an emergency/trauma ward. Electronic alerts for AKI are active on both wards and use changes in creatinine to detect AKI according to the Kidney Disease Improving Global Outcomes criteria. ${ }^{2}$

We aimed to introduce the use of the AKI prediction score into the preoperative assessment of patients undergoing orthopaedic surgery in the Perth Royal Infirmary aiming for $80 \%$ compliance, alongside an intervention bundle for those identified as high risk. The intervention bundle included guidance on perioperative fluid management, withholding certain medications associated with an increased risk of AKI at the time of surgery and on monitoring renal function postoperatively (see online supplementary appendix $1)$. We introduced the tool simultaneously into the preassessment clinics for patients undergoing elective surgery and the orthopaedic wards for patients requiring emergency surgery.

\section{BACKGROUND}

AKI is common postoperatively though reported rates vary depending on the type of surgery. Rates of AKI in our population are between $7 \%$ and $11 \%$ for patients undergoing orthopaedic surgery. ${ }^{1}$

Evidence shows that even mild episodes of AKI are associated with an increased risk of death ${ }^{134}$ and of progression to chronic kidney disease in the long term. ${ }^{5}$ In addition, 
patients who develop AKI spend an average of 4.7 days more in hospital and it is estimated that it costs the National Heath Service (NHS) between $£ 434$ million and $£ 620$ million per year. ${ }^{6}$

There are a number of scoring tools available that predict AKI in a range of settings. ${ }^{7-9}$ The scoring tool we used was specifically developed and validated for use in patients undergoing orthopaedic surgery.

The cause of AKI in the perioperative setting is often multifactorial but there are several interventions with the potential to reduce risk. ${ }^{10}$ Certain commonly prescribed medications are well established in the cause of $\mathrm{AKI}^{11}$ particularly in the context of an acute insult such as hypotension or volume depletion. These include ACE inhibitors, ARBs, non-steroidal anti-inflammatory drugs (NSAIDs) and diuretics. We therefore advised that these medicines were withheld perioperatively in those patients at high risk of AKI. Hypovolaemia and hypotension are also important causes of AKI in the perioperative period and so we advised careful fluid balance monitoring and the prescribing of intravenous fluids if required postoperatively.

Delays in the diagnosis of AKI are associated with poorer outcome. ${ }^{12}$ We included postoperative monitoring of renal function as part of our intervention for those at high risk of AKI.

\section{BASELINE MEASUREMENT}

The ultimate goal of introducing the preoperative AKI prediction scoring tool was to reduce the rate and severity of AKI in patients undergoing orthopaedic surgery at the Perth Royal Infirmary. The power required to generate a clinically and statistically significant result, however, was beyond the scope of this project.

We carried out a baseline audit of current practice by retrospectively reviewing the notes of all patients who had orthopaedic surgery between 6 June 2016 and 19 June 2016. Data were obtained for 37 patients and the information we obtained informed our interventions.

The first objective of this project was to introduce the AKI scoring tool into the preoperative assessment of patients undergoing orthopaedic surgery using an electronic tool that was available on desktop computers and smartphones and to assess if it was reliable and easy to use. At the time of the baseline audit, the AKI scoring tool had not been implemented and so patients were not being risk-stratified.

For patients found to be at high risk of AKI, which we defined as preoperative risk of AKI of greater than $10 \%$, we created a bundle of three interventions aimed at reducing the incidence and/or severity of AKI. We chose a relatively low threshold as the cut-off for high risk based on the simplicity and minimal risk of harm of the proposed interventions as well as the number of patients affected based on our previous work. Our second objective was to alter the management of patients at high risk of AKI according to our intervention bundle.
The first intervention was aimed at ensuring patients were kept well hydrated by keeping an accurate fluid balance perioperatively and considering intravenous fluids if the patient's oral intake was poor or there were any signs of dehydration/hypovolaemia. There was also additional information in the bundle as to when to consider urgent fluid resuscitation. Our measures included whether adequate fluid balance monitoring covering intake and output was in place and if patients who developed hypotension were reviewed. In the baseline audit, only 23/37 (62\%) of patients had adequate fluid balance monitoring postoperatively. Intravenous fluids were prescribed in 16/37 (43\%) patients and 10/17 (59\%) patients were considered high risk for AKI using the scoring tool. Postoperative hypotension (defined as systolic blood pressure (BP) $<100 \mathrm{~mm} \mathrm{Hg}$ ) occurred in nine (24\%) patients. Of these, eight patients had intravenous fluid administered but there were only two cases where the hypotension prompted a documented review. Of note, we did not recommend that all patients at high risk of AKI be given intravenous fluid routinely postoperatively.

Consideration was also given as to whether patients at high risk should be catheterised routinely. Due to the risk of catheter-related infection, we did not include routine catheterisation of high-risk patients.

Our second intervention related to withholding certain medications around the time of surgery. The perioperative drug management guideline in place prior to this project stipulated patients on ACE inhibitors or ARBs should have these medications withheld on the day of surgery and that patients on NSAIDs should stop these 3 days before surgery. It also stated that aldosterone antagonists should be withheld on the day of surgery but not did comment on loop or thiazide diuretics.

In the baseline audit, six patients were on an ACE inhibitor or ARB. All six patients had these medications withheld on the day of surgery though it was restarted on day 1 postoperatively in four patients. Of note, four of the patients who had their ACE inhibitors or ARBs restarted postoperatively required further doses to be withheld during their admission due to low BP, which suggested that restarting these medications routinely on day 1 postoperatively was premature. We recommended that patients at high risk of AKI withhold these medicines on the day of surgery and day 1 and day 2 postoperatively.

With regards to NSAIDs, 4/37 patients were on regular NSAIDs preoperatively. Only one of these patients did not have their NSAIDs withheld preoperatively. This was a patient undergoing palliation for metastatic cancer. NSAIDs were started for postoperative pain in three patients (all low risk). Our intervention bundle did not alter the advice to withhold the NSAIDs from 3 days preoperatively but added that they should be used with caution postoperatively for those at high risk of AKI.

No patients in the baseline audit were on loop or thiazide diuretics; one patient was on spironolactone and 
this was withheld on the day of surgery. Our intervention bundle advised that all diuretics should be withheld routinely on the day of surgery and that fluid balance should be monitored closely when restarting these medications postoperatively. It was agreed that the anaesthetist would make this decision based on the individual patient.

In the baseline audit, 7/37 patients did not have postoperative bloods checked, including one patient who would have been considered at high risk of AKI using the scoring tool. In our intervention bundle, we advised that all patients should have their bloods checked on day 1 and day 3 postoperatively (or on day 2 if they were to be discharged that day) with further monitoring of renal function if the patient develops an AKI. This was based on a separate project in the orthopaedic unit, which reviewed the results of six patients over the age of 75 years who developed an AKI. This found that checking bloods only on day 1 postoperatively would have missed AKI in five out of six cases.

We planned to collect data by reviewing the notes of all patients discharged on selected days and assessing compliance with our intervention.

\section{DESIGN}

Our first objective was that at least $80 \%$ of patients undergoing orthopaedic surgery at the Perth Royal Infirmary (excluding day cases) had their AKI risk calculated using the risk score we previously developed. ${ }^{1}$ Our first step was to incorporate the score into a platform that would be easily accessible for everyone. Using the free platform calcapp.net we created an electronic tool which functioned as an app on smartphone or tablet (iOS or android) or as a web page on a computer. The calculator could be opened on a tablet browser with a link or saved to the desktop to function as an app. Saving it allowed it to be used offline. This allowed easy access for staff. We ensured that it was available on a desktop in case staff did not have access to a smartphone or found it easier to use on a computer. We anticipated that many of the staff using the score would not have prior experience calculating ASA grades and so we included clear guidelines on how to grade patients adapted from the American Association of Anaesthesiology website into our electronic tool.

The calcapp platform ${ }^{13}$ is a web platform to allow the building and hosting of calculator apps without the need for specialist coding experience. It uses the same inputs and formulae as a spreadsheet. The lack of specialist coding knowledge was beneficial as our team had limited prior experience in app design and coding.

We implemented our change in practice for emergency and elective patients simultaneously as the interventions were the same in both groups.

A renal consultant, renal registrar and three anaesthetists with an interest in orthopaedic surgery and AKI were closely involved in designing the intervention. This included planning the baseline audit and using the information gathered from it to create the intervention bundle. Our team also included three Foundation Year 2 (FY2)s; one developed the electronic tool and the other two were involved in data collection.

Prior to implementing the intervention, we consulted various teams and individuals whose support would be crucial to the success of the project. This included the orthopaedic consultants and senior trainees, the wider anaesthetic team and the junior doctors on the orthopaedic wards. We also met with the charge nurses of the orthopaedic wards who disseminated our plan to the ward nurses.

A poster was created with the intervention bundle along with advice on how to carry out a fluid review and when to consider urgent fluid resuscitation. The poster contained a QR code which linked to the electronic tool. Posters were placed in the orthopaedic wards and in the preassessment clinic. The intervention bundle was also available by accessing the electronic tool. The ward clerk in the preassessment unit ensured that there was an adequate supply of high risk of AKI stickers in the preassessment unit and on both orthopaedic wards.

All elective patients are seen in the preassessment clinic by preassessment nurses and have their bloods checked. We met with the preassessment nurses before implementing the intervention to get their feedback and ensure they knew how to use the scoring tool. Our plan was that patients would have their AKI score completed in the preassessment clinic and documented in the medical notes. Patients who are high risk would get a red sticker added to the front of their notes stating 'High risk of AKI'. This would be seen by the secretaries who would add a note to the operating theatre list flagging that the patient was at high risk of AKI. As the score requires a patient's eGFR, it cannot be done until the following day. While this could potentially cause problems as patients may need to be contacted after the clinic, it would only affect patients on loop or thiazide diuretics and the preassessment nurses did not think this would significantly add to their workload. The admitting junior doctor would then be responsible for making the postoperative medication changes and ensuring bloods are checked postoperatively. The stickers highlighting those patients at high risk would also be visible to the nursing staff reinforcing the need for accurate fluid balance monitoring postoperatively (though this would be expected in all patients).

Emergency patients are admitted directly from Accident \& Emergency and are clerked by the junior doctors. For these patients the clerking doctors would have responsibility for completing the score, putting the sticker and intervention bundle in the notes if indicated, and making any medication adjustments. Again, the ward staff would be responsible for the remainder of interventions.

\section{STRATEGY}

We used Plan, Do, Stay, Act (PDSA) cycles to achieve our first objective which was that at least $80 \%$ of patients undergoing orthopaedic surgery requiring an overnight 


\begin{tabular}{|c|c|c|c|c|}
\hline PDSA cycle & 1 & 2 & 3 & 4 \\
\hline Aim & $\begin{array}{l}\text { Establish reliability of } \\
\text { scoring tool and accuracy } \\
\text { completing the AKI risk } \\
\text { score }\end{array}$ & $\begin{array}{l}\text { Improve accuracy } \\
\text { completing the AKI risk } \\
\text { score }\end{array}$ & $\begin{array}{l}\text { Improve compliance with } \\
\text { completing the AKI risk } \\
\text { score }\end{array}$ & $\begin{array}{l}\text { Assess compliance with } \\
\text { intervention bundle }\end{array}$ \\
\hline Intervention & $\begin{array}{l}\text { AKI risk score introduced } \\
\text { with electronic tool for } \\
\text { calculating it on desktop } \\
\text { and mobile devices } \\
\text { available along with an } \\
\text { intervention bundle for } \\
\text { patients at high risk of AKI }\end{array}$ & $\begin{array}{l}\text { Coincided with junior } \\
\text { doctor change-over. } \\
\text { Face-to-face education } \\
\text { session with new } \\
\text { doctors, highlighted } \\
\text { guidance in scoring } \\
\text { tool on ASA grading } \\
\text { to ensure accuracy } \\
\text { completing the score }\end{array}$ & $\begin{array}{l}\text { Junior and senior } \\
\text { orthopaedic staff briefed } \\
\text { at daily trauma meeting } \\
\text { with demonstration of } \\
\text { scoring and explanation } \\
\text { of bundle rationale }\end{array}$ & $\begin{array}{l}\text { Collect data on larger } \\
\text { sample. Presentation } \\
\text { of preliminary results at } \\
\text { department audit meeting } \\
\text { and ongoing verbal } \\
\text { encouragement given to } \\
\text { junior staff }\end{array}$ \\
\hline Outcome & $\begin{array}{l}\text { Three of four patients had } \\
\text { score completed, correct } \\
\text { in only one patient. Errors } \\
\text { due to incorrect ASA } \\
\text { grade being used }\end{array}$ & $\begin{array}{l}\text { Drop in compliance } \\
\text { completing the score } \\
(2 / 6) . \text { The score was } \\
\text { completed accurately }\end{array}$ & $\begin{array}{l}\text { Significant improvement } \\
\text { with } \\
10 / 13 \text { patients scored } \\
\text { having score complete; } \\
9 \text { of those scored were } \\
\text { accurate }\end{array}$ & $\begin{array}{l}12 / 18 \text { patients scored. } \\
\text { All scores accurate and } \\
\text { stickers applied to all high- } \\
\text { risk notes }\end{array}$ \\
\hline
\end{tabular}

AKI, acute kidney injury; ASA, American Society of Anaesthesiologists.

stay would have their AKI risk calculated using the scoring tool (see table 1).

PDSA cycle 1 aimed to assess if the scoring tool was functional, reliable and being completed accurately. The staff in the preassessment clinic and on the orthopaedic wards were advised to start using the AKI risk score and intervention bundle. Data were collected covering admissions between 29 June 2017 and 18 July 2017 and focused on whether the score was being completed accurately. Only four patients were identified in our sampling; data were also collected on day case and non-operative patients but as the score is not intended for use in these patients they were excluded. Three out of four patients had the score completed but unfortunately it was done incorrectly on two occasions (both on emergency patients). This was most likely due to the incorrect ASA grade being used in both cases. Verbal feedback from staff confirmed that the electronic tool was reliable and functioning well.

In the second PDSA cycle, we aimed to improve the accuracy of scoring. This cycle coincided with a change in the junior doctors on the wards; a face-to-face education session was held with the new doctors, which covered the rationale for the intervention and their role. The guidance from the American Society of Anaesthesiology on ASA grading included in the scoring tool, was highlighted to them. This cycle collected data covering admissions between 12 August 2017 and 31 August 2017. Data were collected on six patients; unfortunately, only two of these patients had the score completed (both accurate). This may have been related to the fact that the new doctors starting on the ward had a lot of new processes to adapt to and did not prioritise completing the score.
The main aim of the third PDSA cycle was to boost compliance with completing the score. Verbal reminders were given to the doctors at the trauma meeting (attended by all senior and junior orthopaedic staff). This included a practical demonstration on completing the score and explanation of the rationale behind the intervention bundle. Data were collected covering admissions between 1 September 2017 and 28 September 2017. Reassuringly there was a significant boost in compliance with the score being carried out in 10 out of 13 patients who had their notes assessed. This was largely driven by elective patients with 9 out of 10 elective patients having the score complete compared with only 1 out of 3 emergency patients. The score was accurate in 9 out of 10 cases.

The fourth PDSA cycle covered patients admitted between 2 October 2017 and 28 October 2017 with the focus in this cycle being to improve compliance with completing the score and to collect a larger sample of data to assess how well the intervention bundle was being adhered to in high-risk patients. Preliminary results were presented at the departmental audit meeting and ongoing verbal encouragement was given to medical staff. Twelve out of 18 patients in this cycle had the score completed $(10 / 14$ elective patients and $2 / 4$ emergency patients). The score was accurate in all cases. Eight of the patients were high risk, all of whom had stickers appropriately placed on the front of their notes. A full induction is planned for the new doctors in the next rotation and a rolling departmental audit will continue. 


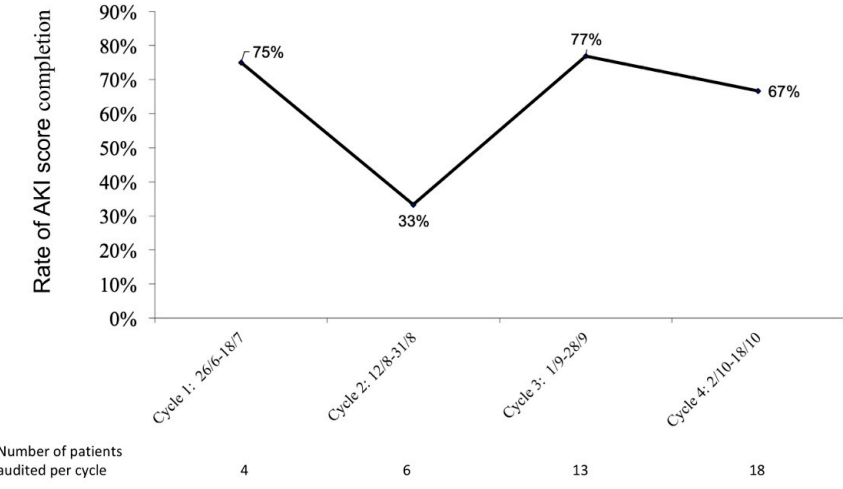

Figure 1 Compliance with using AKI risk score over four PDSA cycles. AKI, acute kidney injury.

\section{RESULTS}

In total, the score was completed in $27 / 41$ (66\%) patients who had their notes reviewed. There were relatively small numbers in the first and second PDSA cycles but there was a drop in compliance in the second PDSA cycle that we think is at least partly attributable to new doctors starting on the ward (it was the first rotation for the Foundation Year 1 (FY1 doctors). Overall compliance improved again following this (see figure 1).

There was a higher rate of compliance with the AKI scoring tool in elective patients; the combined results from the third and fourth PDSA cycles found the rate of compliance with the score was just below target at 19/24 $(79 \%)$. The corresponding result for emergency patients was disappointingly lower at three of seven $(43 \%)$. Of note; for patients undergoing either hip or knee surgery the compliance was better at 25/32 (78\%) compared with $2 / 9(22 \%)$ for patients undergoing upper limb or foot surgery.

The accuracy of using the tool significantly improvement as the project progressed and was $100 \%$ by the fourth PDSA cycle.

We also collected data to assess compliance with the intervention bundle. Twelve of the patients who had their score calculated were classified as high-risk; there was $100 \%$ compliance with using the high-risk of AKI stickers for these patients. We calculated the scores retrospectively for patients who did not have them done preoperatively; 4/14 would have been classified as high-risk.

Fluid balance monitoring covering intake and output was adequate in 9/12 (75\%) of those patients classified as high-risk. Reassuringly, our intervention did not adversely impact those patients classified as low-risk where a similar rate $(11 / 15,73 \%)$ had adequate fluid balance monitoring. We did observe however poorer monitoring in those with no score done where only 7/14 (50\%) had adequate fluid balance monitoring. The lower rate in the latter group may be in part due to a higher proportion of patients undergoing upper limb or foot surgery $(7 / 14)$ as ward staff may not prioritise these patients for close monitoring postoperatively. The overall rate of adequate fluid balance monitoring was $27 / 41(66 \%)$, very similar to the $62 \%$ in the baseline audit.

Five patients developed postoperative hypotension (systolic BP $<100 \mathrm{~mm} \mathrm{Hg}$ ); only one patient was reviewed and given intravenous fluid (they had been scored as low-risk). We advised that hypotension in any patient should prompt a medical review and intravenous fluid should be considered. This has not demonstrably changed practice.

With regard to medication changes for patients at high risk of AKI; seven patients were on an ACE inhibitor or ARB and six were on diuretics. All were withheld appropriately. The preoperative advice regarding NSAIDs was the same in all patients regardless of AKI risk. Three patients classified as being at high risk of AKI were on NSAIDs preoperatively; they were withheld in two of them. Similarly, two of three patients found to be at low risk of AKI and two of four patients who did not get the score done had their NSAIDs withheld preoperatively. Two of 12 patients at high risk of AKI were given NSAIDs postoperatively for pain management.

Bloods were checked as per our intervention bundle (ie, at least twice postoperatively) in all patients found to be at high risk of AKI. Bloods were checked at least once in $24 / 29(83 \%)$ of the remaining patients. Of the five patients who did not have postoperative bloods checked, one was at high risk but did not have the score done.

Two of the patients developed AKI postoperatively; both would have been classified as high risk of AKI preoperatively though the score was not completed in one of the patients.

\section{LESSONS AND LIMITATIONS}

One of the strengths of our project is that it is generalisable to other hospitals. The score we used has been externally validated for use in patients undergoing orthopaedic surgery. We involved the key stakeholders from the beginning of our project and had strong support from both the anaesthetic and orthopaedic teams.

A limitation of this study was the small numbers of patients in the first and second PDSA cycles. This was in part because some of the data we collected were from patients not affected by our intervention so were excluded from our analysis. The data were collected by an FY2 working in the orthopaedic department. We limited bias by selecting certain days to collect the data and reviewing the notes of all patients discharged that day. As the score was completed on admission this gave us a spread of admission days to assess and limited the effect one or two individuals may have had.

We anticipated that the ASA grade would be new to many of the staff completing the score. We therefore included additional information within our scoring tool. Accuracy with the score improved as experience increased.

We introduced the score simultaneously for elective patients and emergency patients. Although the score is being used well for elective patients it remains 
disappointingly low for emergency patients. This is, in part, driven by very low rates of score completion for patients undergoing upper limb or foot surgery. In retrospect, a two-stage approach, implementing our project for elective patients first followed by emergency patients later, may have allowed us to target our interventions with greater effect.

There was still relatively poor fluid balance monitoring of orthopaedic patients postoperatively. As this applies to all patients, not just those at high risk, it may be that this is better taken forward as a separate quality improvement project.

\section{CONCLUSION}

We introduced a preoperative scoring tool that predicts a patient's risk of postoperative AKI into the assessment of patients undergoing orthopaedic surgery that require an overnight stay. Use of the score was good among patients undergoing elective surgery but remained significantly below our target for patients admitted for emergency procedures.

We were able to demonstrate that our electronic scoring tool was reliable and could be used accurately. There was excellent compliance with medication adjustments and postoperative renal function monitoring in patients found to be at high risk of AKI, but postoperative fluid balance monitoring was still relatively poor.

There will be a rolling audit in the department that will aim to further improve compliance with using the score. We also plan to implement our scoring tool into the orthopaedic departments at the other hospitals within NHS Tayside.

Contributors DPB was involved in the collection of the baseline data, developing the intervention bundle and liaising with the relevant teams prior to implementation. He was also responsible for writing the first draft of the manuscript and is responsible for overall content as guarantor. SB was responsible for developing and validating the scoring tool and conceived the idea of using it alongside an intervention bundle to reduce the risk of AKI. She also contributed to the development of the intervention bundle. EW was involved in collecting the baseline data for the project while FR was responsible for collecting the data following the introduction of the scoring tool and intervention bundle. KG was responsible for developing the electronic tool that allowed the score to be used on smartphones and desktop computers. CB, RV and SS all contributed to the design of the baseline data collection and the development of the intervention bundle. All authors critically revised the manuscript for important intellectual content, gave their final approval of the version to be published and agree to be accountable for all aspects of the work.

Funding The authors have not declared a specific grant for this research from any funding agency in the public, commercial or not-for-profit sectors.

Competing interests None declared.

Patient consent for publication Not required.

Provenance and peer review Not commissioned; externally peer reviewed.

Open access This is an open access article distributed in accordance with the Creative Commons Attribution Non Commercial (CC BY-NC 4.0) license, which permits others to distribute, remix, adapt, build upon this work non-commercially, and license their derivative works on different terms, provided the original work is properly cited, appropriate credit is given, any changes made indicated, and the use is non-commercial. See: http://creativecommons.org/licenses/by-nc/4.0/.

\section{REFERENCES}

1. Bell S, Dekker FW, Vadiveloo T, et al. Risk of postoperative acute kidney injury in patients undergoing orthopaedic surgery-development and validation of a risk score and effect of acute kidney injury on survival: observational cohort study. BMJ 2015;351:h5639.

2. Kellum JA, Aspelin P, Barsoum RS, et al. KDIGO Clinical Practice Guideline for Acute Kidney Injury. Kidney Int Suppl 2012;2:1-138.

3. Bucaloiu ID, Kirchner HL, Norfolk ER, et al. Increased risk of death and de novo chronic kidney disease following reversible acute kidney injury. Kidney Int 2012;81:477-85.

4. Linder A, Fjell C, Levin A, et al. Small acute increases in serum creatinine are associated with decreased long-term survival in the critically ill. Am J Respir Crit Care Med 2014;189:1075-81.

5. Coca SG, Singanamala S, Parikh CR. Chronic kidney disease after acute kidney injury: a systematic review and meta-analysis. Kidney Int 2012;81:442-8.

6. Taylor J. Acute Kidney Injury: calculating the cost. Heal Serv J Suppl 2011:1.

7. Palomba $\mathrm{H}$, de Castro I, Neto AL, et al. Acute kidney injury prediction following elective cardiac surgery: AKICS Score. Kidney Int 2007;72:624-31.

8. Mehran R, Aymong ED, Nikolsky E, et al. A simple risk score for prediction of contrast-induced nephropathy after percutaneous coronary intervention: Development and initial validation. J Am Coll Cardiol 2004;44.

9. Grimm JC, Lui C, Kilic A, et al. A risk score to predict acute renal failure in adult patients after lung transplantation. Ann Thorac Surg 2015;99:251-7.

10. Goren O, Matot I. Perioperative acute kidney injury. Br J Anaesth 2015;115:ii3-ii14.

11. Bellomo R, Kellum JA, Ronco C. Acute kidney injury. Lancet 2012;380:756-66.

12. Yang L, Xing G, Wang L, et al. Acute kidney injury in China: a crosssectional survey. Lancet 2015;386:1465-71.

13. Neosupport. Calcapp. https://www.calcapp.net (accessed 14 Feb 2019). 